\title{
Evaluation of Time and Frequency Domain-Based Methods for the Estimation of Harmonics-to-Noise-Ratios in Voice Signals
}

\author{
Carlos A. Ferrer, Eduardo González, and María E. Hernández-Díaz \\ Center for Studies on Electronics and Information Technologies, Central University of Las \\ Villas, Zip code: 54830, Santa Clara, Cuba \\ \{cferrer, moreira, mariae\} @uclv.edu.cu
}

\begin{abstract}
In this paper several approaches of time and frequency domain-based algorithms to estimate harmonics-to-noise ratios (HNR) in voice signals are compared. The approaches covered incorporate a recent time-domain correction to a classic method, as well as a frequency-domain adjustment introduced here. The experimental comparisons include the number of pitch periods needed to obtain the best HNR estimates, as well as the sensitivity of the methods to different perturbations of the periodicity pattern, like shimmer, jitter, noise and combinations of them. Time domain methods show better performance than frequency-based approaches, and moreover, the correction to the ensembleaverage time domain technique reduces the required number of pulses by an order of magnitude.
\end{abstract}

\section{Introduction}

It has been widely reported that estimates of additive noise levels in voice signals are of diagnostic value to several speech pathologies ([20], [8], [4], [18]). The determination of Harmonics-to-Noise Ratios (HNR) in voiced speech signals is intended to yield a measure of additive noise in the acoustic waveform. For this purpose, several methods have been proposed [11], both in time and frequency (or transformed) domain, and there is no definitive satisfactory solution. A most troubling issue is the interaction of other perturbations of the periodicity pattern, like pulse duration variability (jitter) and pulse amplitude variability (shimmer), in the measured HNR [5]. According to the derivations described analytically in [17], it is not possible to perform separate measurements of each type of perturbation by using spectralbased methods. On the other hand, time domain methods have been criticized ([6][15]) for depending on the correct determination of the individual pulse (pitch epoch) boundaries, among many other method-specific factors.

A much referenced time-domain method was proposed by Yumoto et al. [20], who introduced the finding of a pitch epoch "template" by averaging the ensemble of individual pitch pulses. The averaged waveform is known to present a noise variance reduced by a factor of $N$, the number of averaged pulses. Yumoto proposed to measure HNR as the ratio of the variance of the template to the variance of the differences of the individual pulses with the template (see equation 5). It has been 
criticized [7] for the need of a relatively large number of pulses (30-50) to effectively remove the noise from the template, and influences of jitter and shimmer in the resulting measures of HNR [5].

In spite of this, Yumoto's method remains “the most commonly used time-domain technique" [11] and several authors have worked on its improvement. In [1], a singlepass method was proposed which accounted, under certain considerations, for jitter, shimmer and offset effects. Later, Dynamic Time Warping [13] and Zero Phase Transforms [14] of individual pulses prior to the averaging were used to reduce waveform variability influences in the template. For the same purpose, the ensemble averaging technique was applied [11] to the spectral representations of individual glottal source pulses. Dealing again with waveform variability, Functional Data Analysis was used [9] to perform an optimal time-alignment of pulses prior to averaging. Recently, a theoretical formula (see equation 6) was derived to correct the ensemble averages estimates of HNR for any number of pulses [3], suppressing the need of using a large $N$.

The objective of this paper is to address two issues that remain unclear. First, the ability of the Ensemble-Averages correcting formula in [3] to actually determine the HNR for any number of pulses is tested using simulated signals. Second, the practical extent to which the frequency-domain HNR estimation methods are affected by jitter and shimmer perturbations. The validity of the results in [3] is tested for different pulse lengths, in samples, allowing a more realistic understanding of its practical usefulness. The method in [3] is also compared to the original time-domain version [20] and a correction (developed in this paper) to a frequency domain method [8][2].

\section{Description of Signals, Methods and Experiments}

\subsection{Signals}

Simulated vowels were generated according to the method used in [10] and [12], where the speech signal $s(t)$ is obtained as the convolution of two signals, the vocal tract impulse response $h(t)$ and an excitation impulse train $i(t)$ :

$$
\begin{gathered}
i(t)=\sum_{n} k\left(t_{n}\right) \delta\left(t-t_{n}\right) \\
h(t)=\sum_{m=1}^{M} a_{m} e^{-b_{m} t} \cos \left(2 \pi f_{m} t\right) \quad(t \geq 0)
\end{gathered}
$$

This convolution results in:

$$
s(t)=\sum_{n} k\left(t_{n}\right) h\left(t-t_{n}\right)
$$

where $t_{n}$ is the time instant of the $n$-th excitation and $k$ is its amplitude. The terms $a_{m}$, $b_{m}$ and $f_{m}$ represent the amplitude, bandwidth and central frequency, respectively, of the $\mathrm{M}$ resonators (formants) used to model the vocal tract. The values of $a_{m}, b_{m}$ and $f_{m}$ used for these resonators are the same as in [10] and [12], corresponding to a vowel $/ \mathrm{a} /$ and $\mathrm{M}=5$. 
The use of equations (1) and (2) to synthesize the vowel yields an easy way to vary the amount of jitter by controlling $t_{n}$, while $k$ can be used to vary shimmer, and the HNR can be controlled by adding noise to $s(t)$.

The sampling frequency $F_{S}$ was set to $22.05 \mathrm{kHz}$, and the mean value of fundamental period T0 is $t_{m}=1 / 150$ seconds ('mean' fundamental frequency $\mathrm{F} 0=150$ $\mathrm{Hz}, t_{m}=147$ samples). These values for $F s$ and F0 are the same than in [12]. For simplicity reasons, only the closest two precedent impulses to the current " $t$ " were considered in the synthesis of (2), since $h(t)$ decays to negligible values for $3 * t_{m}$. In any case, a certain amount of interference between two consecutive pulses is introduced. The length of the generated speech signals was set to two seconds, which gives, together with the value of $t_{m}$ used, an average of 300 pitch pulses on each signal. Four types of signals were generated, according to the presence of jitter, shimmer, additive noise, or a combination of the three periodicity perturbations.

Jittered Signals: The amplitude factor of equations (1) and (2) is left constant $(k=1)$ and no noise is added to the signal $s(t)$. The impulse excitation instants are obtained as $t_{n}=t_{n-1}+t_{m}+u(n)$, where $u(n)$ is a random real value uniformly distributed in the interval $\pm u_{m}$. The time difference between two adjacent excitation instants will have then a uniform probability distribution in the range $t_{m} \pm u_{m}$. The average pitch duration in samples is $t_{m}=147$. Different values for $u_{m}$ were used, varying from 0 to 35 , in steps of 5 samples, corresponding to values from 0 to $23.8 \%$ of $t_{m}$ in steps of $3.4 \%$. According to [10] jitter almost never exceeds the $25 \%$ of $t_{m}$.

Shimmered Signals: The amplitude $k(n)$ of the impulses used to generate $s(t)$ are obtained as: $k(n)=1+v(n)$, where $v(n)$ is a random real value, uniformly distributed in the interval $\pm v_{m}$. The values of $v_{m}$ used were twice the values of $u_{m}$ in the previous experiment, measured in percent of the unaltered amplitude $k=1$, going from 0 to $47.6 \%$ in steps of 6.8 . This relationship was chosen to keep the ratio of usually accepted limits of jitter (25\%) [10] and shimmer (50\%) [19]. The temporal separation between the pulses was kept constant, equal to $t_{m}$. No noise was added to $s(t)$.

Additive Noise Only: A clean signal $s(n)$, obtained for constant values of $k=1$ and $t_{n+1}=t_{n}+t_{m}$, was contaminated with additive white gaussian noise $e(n)$, such that the ratio of the variances of $s(n)$ and $e(n)$ met an intended value of $\mathrm{HNR}$ in $\mathrm{dB}$. The values of HNR used (in $\mathrm{dB}$ ) were $\infty, 22,18,15,12,8,5$, and 2 .

Combined Perturbations: All the parameters ( $u_{m}, v_{m}$ and HNR) were varied simultaneously, in ascending order of perturbation, in the same amounts as in the three previous experiments. In this way, eight different cases are obtained, ranging from the perfectly periodic $s(t)$ to the most distorted waveform with $u_{m}=23.8 \%$, $v_{m}=47.6 \%$ and $\mathrm{HNR}=2 \mathrm{~dB}$.

\subsection{HNR Estimation Methods}

The HNR estimation methods compared in this paper are the original ensemble averaging technique [20] and its correction to any number of pulses [3] in the time domain. In the frequency domain, the approach proposed in [8] and generalized in [2] was chosen. A brief description of the methods follows. 


\section{Time-Domain Method}

The ensemble averaging technique departs from assuming each pulse representation $x_{i}(t)$ prior to averaging as a repetitive signal $s(t)$ plus a noise term $e_{i}(t)$ :

$$
x_{i}(t)=s(t)+e_{i}(t)
$$

A "template" of the periodic component is obtained as the average of the $N$ individual pulses:

$$
\bar{x}(t)=\frac{\sum_{i=1}^{N} x_{i}(t)}{N}=s(t)+\frac{\sum_{i=1}^{N} e_{i}(t)}{N}
$$

and an expression to estimate the HNR is proposed as:

$$
H N R_{\text {Yum }}=\frac{N \times E\left[\bar{x}^{2}(t)\right]}{\sum_{i=1}^{N} E\left[\left(x_{i}(t)-\bar{x}(t)\right)^{2}\right]}
$$

with $\mathrm{E}[]$ denoting the expected value operation. In 3 it is shown that, if noise and signal are uncorrelated, the actual HNR can be expressed in terms of (5) as:

$$
H N R=\frac{N-1}{N} H N R_{Y u m}-\frac{1}{N}
$$

\section{Frequency-Domain Method}

The frequency-domain approaches are mostly based in the calculation of a Discrete Fourier Transform to a segment of the signal containing several pulses. Amongst the many transformed-domain approaches ([2][4][6][7][8][11][15][16]) the one proposed by Kojima [8] was chosen, since it shares many of their limitations, but at least suppresses the windowing effects in the HNR estimation. The basic difference of [8] compared to the others is that the segment of analysis is chosen so that it contains an exactly integer number of pulses $(N)$, regardless of its length in samples $(T)$. This method has not been favored by researchers, preferring values of $T$ multiples of 2 to use FFT algorithms.

Since the signal is assumed quasi-periodic, the harmonic energy should be concentrated every $N$ spectral component, corresponding to the numerator (Num) in (8), while the noise energy can be assumed as the difference of the total energy and the harmonic one, as written in the denominator (Den) of (8). $T$ is the length in samples of the sum of the $N$ pulses.

$$
H N R_{K o j}=\frac{N u m}{D e n}=\frac{\sum_{i=1}^{T / N} X(i * N)^{2}}{\sum_{i=1}^{T} X(i)^{2}-\sum_{i=1}^{T / N} X(i * N)^{2}}
$$


The original approach in [8] used $N=3$ and established spectral bands where the calculated HNR correlated best to the perceived hoarseness. In [2] the method was generalized to use any $N$ and frequency bands.

\section{A Correction to Kojima's Method}

Revisiting the idea behind Kojima's method two corrections are apparent: the numerator overrates the harmonic energy, due to the contribution of the noise present at $X\left(i^{*} N\right)$, which has been neglected, and the denominator underrates the noise energy, because of the removal of the noise at the same frequencies.

Assuming that the noise has a flat power spectral density and limiting the analysis in the spectral domain to a number of bins $B$ equal to the closest multiple of $N$ smaller than $L$, it can be shown that the total noise energy $E_{N}$ in the interval of $B$ bins is:

$$
E_{N}=\operatorname{Den} \frac{N}{N-1}
$$

Similarly, the total harmonic energy $E_{H}$ in the $B$ bins can be expressed as:

$$
E_{H}=N u m-\left(N_{M} * H\right)=N u m-\frac{E_{N}}{B} \frac{B}{N}=N u m-\frac{E_{N}}{N}=N u m-\frac{D e n}{N-1}
$$

where $N_{M}=E_{N} / B$ is the mean noise energy, and $H=B / N$ is the number of harmonics in the $B$ bins. The actual $\mathrm{HNR}=E_{H} / E_{N}$ is then given by:

$$
H N R=\frac{E_{H}}{E_{N}}=\frac{N u m-\frac{D e n}{N-1}}{\operatorname{Den} \frac{N}{N-1}}=\frac{N u m}{\operatorname{Den}} \frac{N-1}{N}-\frac{1}{N}=H N R_{K o j} \frac{N-1}{N}-\frac{1}{N}
$$

The resemblance of (10) and (6) is remarkable, and it suggests both methods to be equivalent. The equivalence of time and spectral domain HNR estimations has also been assumed in [15], although the results favored the time-domain approach. It is evident that the correction in (10) is largely relevant for Kojima's method, where small values of $N$ were used $(N=3)$.

\subsection{Experiments}

The experiments performed were all programmed in MatLab 6.5, and can be separated in two sets:

\section{Time-Domain HNR Sensibility to the Number of Pulses and Pulse Lengths}

In this set of experiments the actual ability of the theoretical derivation in [3] (given by (6)) to correct the results of Yumoto's approach (5) is tested. Theoretically, (6) can correct (5) for any number of pulses $(N)$ given that the noise and the repetitive components, as well as each realization of noise, are uncorrelated. A result of zero correlation in stochastic processes is more probable as the number of samples considered approaches infinity. In practice, pitch pulse duration and sampling frequencies impose limits to the pulse lengths $(L)$. For instance, with a typical 
telephone-quality sampling frequency $(8 \mathrm{kHz})$ a child or an adult woman with moderately high $\mathrm{F} 0=400 \mathrm{~Hz}$ would generate only 20 samples per period. No study of HNR is likely to be conducted under such circumstances, but this example is illustrative in terms of practical low limits of $L$. In this work the original [20] and corrected [3] ensemble averages approaches were tested for values of $N$ between 2 and 50, and $L$ between 30 and 10000 .

The experiment consisted in evaluating (5) and (6) for a set of $N$ pulses generated randomly by adding $N$ random vectors $\boldsymbol{x}_{i}$ of length $L$ to a common random vector $s$ of the same length. The variances of $\boldsymbol{s}$ and $\boldsymbol{x}_{\boldsymbol{i}}$ were chosen to be 4 and 1, respectively, such that calculated HNR should be 4, equivalent to $6 \mathrm{~dB}$. This value is in the range of HNRs tested in the "Additive Noise Only" signals described in section 2.1, allowing the results of this experiment to be extended to those signals.

\section{Sensitivity of HNR Estimation Methods to Different Periodicity Perturbations}

This set of experiments consists in the evaluation of the different methods (both time and frequency-domain based) abilities to estimate HNR in the presence of the perturbations shown by the signals described in section 2.1. The objective in this paper is to explore each method's ability to correctly estimate the HNR, and thus, no band limits were imposed on the frequency domain estimation of HNR. More specific configuration settings are described in section 3. A preliminary experiment is conducted comparing the original and corrected Kojima's frequency-domain method (given by (7) and (10)) in the case of "Additive Noise Only", to illustrate the importance of the correction.

\section{Results and Discussion}

The results of the experiments are divided in the same two sets that were described in section 2.3.

\section{Ensemble-Averages HNR sensibility to Number of Pulses and Pulse Lengths}

The results of the comparison of (5) and (6) for different $N$ and $L$ are shown in Fig. 1. The figure plots the mean absolute differences between actual and obtained HNRs for 1000 realizations of the random variables involved in the experiment. The results are normalized with respect to the actual HNR (HNR=4).

The original HNR estimation method shows the criticized need of large $N$ to reach values closer to the minimum "steady state" errors, which occurs for $N$ in the range from 30 to 50 in correspondence with what is stated in [7]. The plot of corrected HNR can be used to realize the limits of the theoretical "complete" suppression of the effect of $N$ in HNR estimation. It can be seen that the mean "steady state" error is reached for different values of $N$, depending on the number of samples comprised in a pulse length. For a relatively low number of samples (i.e. 30 or 100) a steady error $(22 \%$ and $12 \%$, respectively) is obtained for 8 or 7 averaged pulses. A "medium" sized pulse (300 samples) reaches a steady error from 4 to 7 pulses (from $8 \%$ to $7 \%$ mean errors). No more than 4-5 pulses are needed to reach the steady mean error value when the pulse $L$ is above 300 samples. 


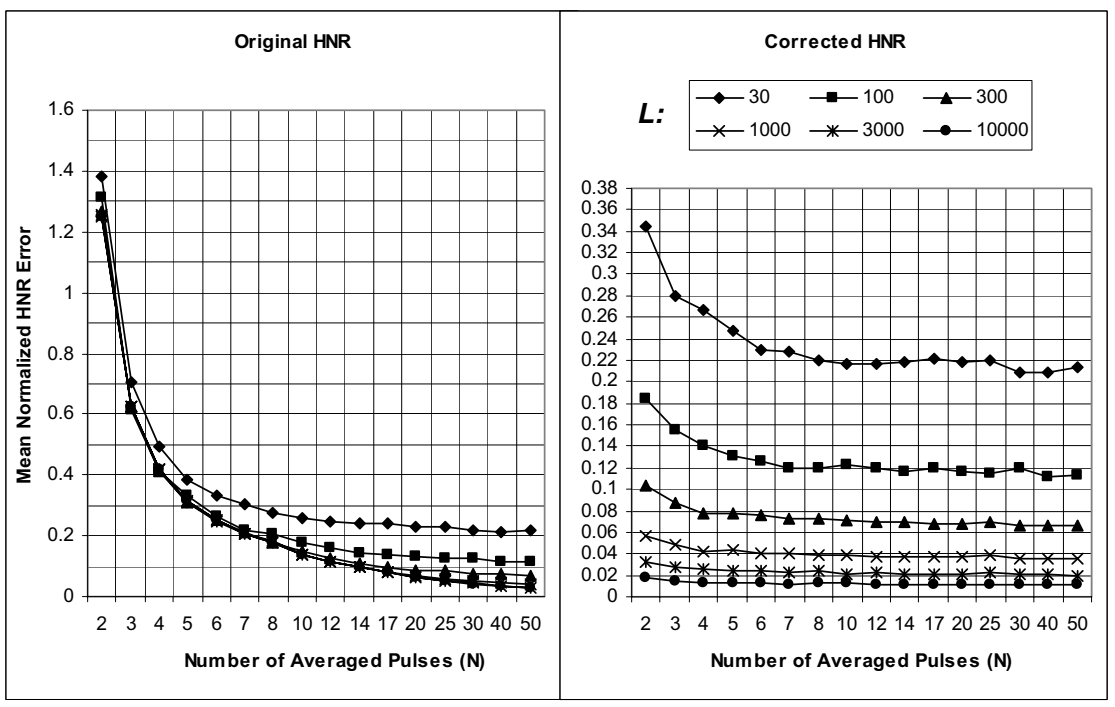

Fig. 1. Original (left) and corrected (right) values of HNR obtained for different cases of averaged pulses $(N)$ and pulse lengths $(L)$

\section{Sensibility of HNR estimation methods to Periodicity Perturbations}

The illustration of the importance of the correction in (10) of the frequency-domain approach in [8] given in (7) is depicted in Fig. 2. Segment sizes were fixed at 3 (original option in [8]) and 7 pulses, to test the influence of the number of pulses. The latter limit was set to avoid the already criticized use of a large $N$.

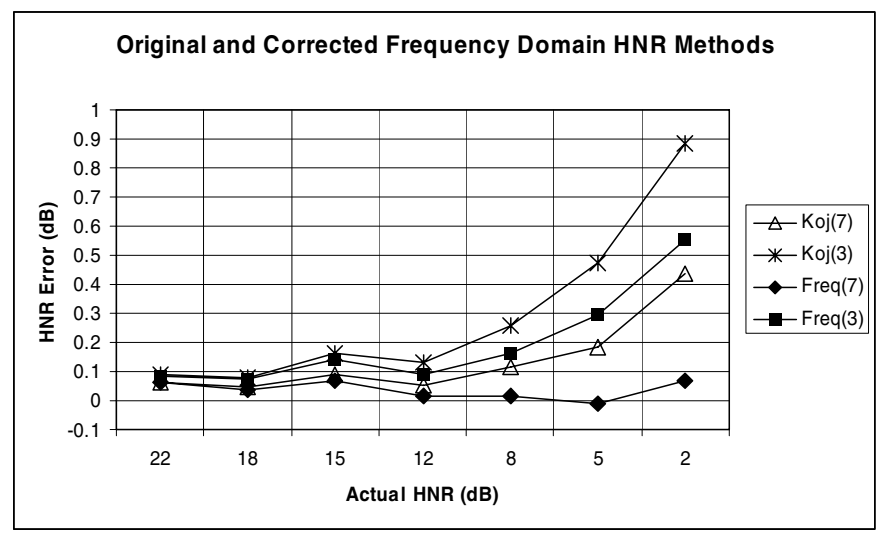

Fig. 2. Comparison of original (Koj) and corrected (Freq) values of HNR in the presence of "Additive Noise Only", calculated for 3 and 7 pulse segments 
These results demonstrate that in the absence of other perturbations of the periodicity pattern, the correction works better for larger $N$. It is also relevant that the effect of changing $N$ from 3 to 7, moving from "Koj(3)" to "Koj(7)", causes a larger error reduction than the one introduced by correcting "Koj(3)" to "Freq(3)". The frequency-domain alternatives compared to the time domain approaches in the other perturbation types are only "Freq(3)" y "Freq(7)".

The signals generated according to the procedure described in section 2.1 were used to evaluate the ability of the different HNR estimation methods to cope with the perturbations present in the signals. Two different $N$ (7 and 50) were considered in the original "Orig" and corrected "Corr" time-domain approaches. The lower limit was set considering the results of the experiment in Fig. 1 and the pulse length $(L)$ of the signals used (147 samples). The results are shown in Fig. 3.

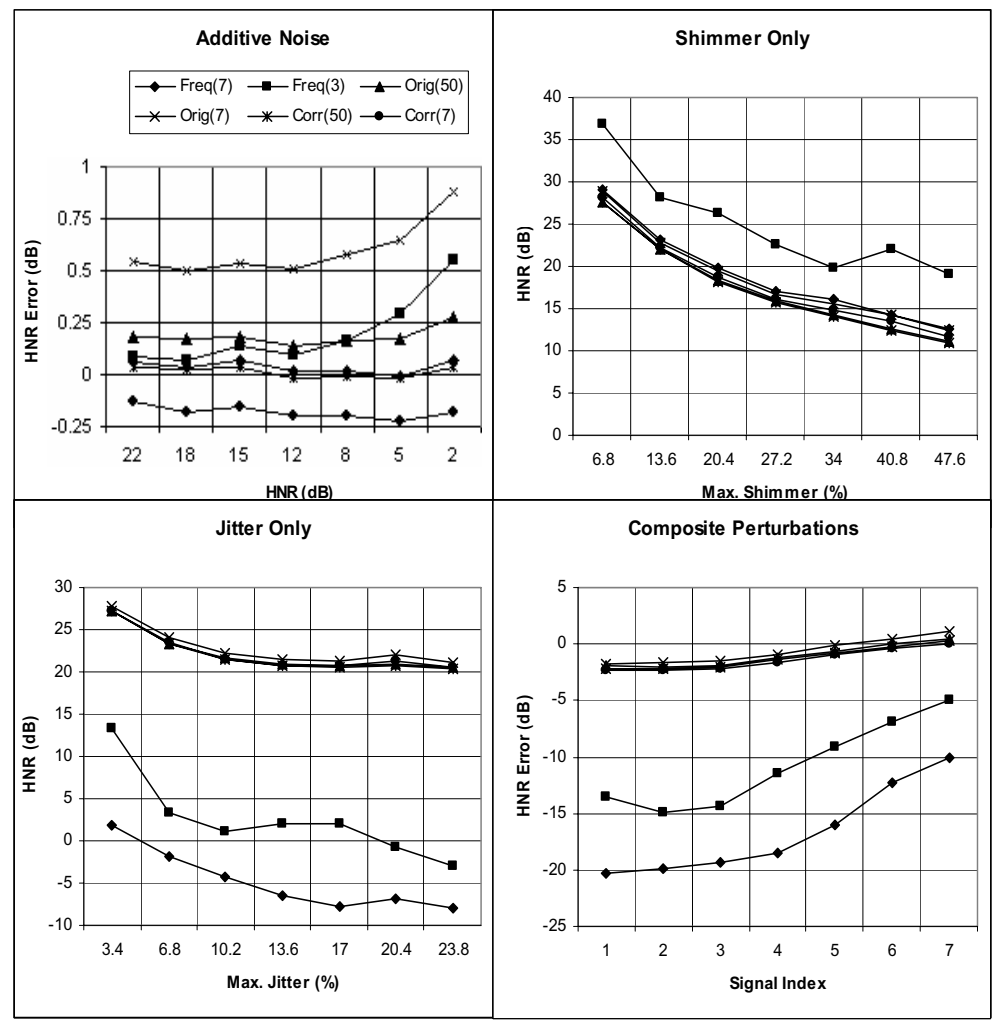

Fig. 3. Results obtained in the experiment on HNR sensibility to periodicity perturbations

The results of the methods facing additive-noise perturbations (upper-left graph in Fig. 3) are demonstrative of their intrinsic ability to measure HNR. An important issue in the time-domain is the similar results of "Corr(7)" and "Orig(50)", showing the importance of using equation (6). The most accurate of the time-domain alternatives is, as expected, "Corr(50)", but "Freq(7)" performs with similar 
exactitude with an $N$ almost an order of magnitude smaller. This indicates that in the absence of jitter and shimmer, the frequency-domain approach performs better.

In the presence of shimmer (upper-right graph in Fig. 3) all the methods show similar deteriorations in the estimated HNR (theoretically infinite), with the exception of "Freq(3)", which is visibly superior. We don't have at this moment an elaborate explanation for this phenomenon. The only guess we can make is that in so few pulses $(N=3)$ the random AM modulation is not so variable to significantly affect the spectrum, but a strict mathematical derivation is not readily available. The coincidence of the time-domain based methods with "Freq(7)" strengthens the idea that all of them are similarly shimmer-sensitive, and the effect in "Freq(3)" should be caused by a calculation bias related to the small value of $N$.

The "Jitter Only" experiment (bottom-left graph in Fig. 3) confirms the predicted [17] sensitivity to jitter of frequency-domain approaches, with estimated HNR (theoretically infinite) more than $15 \mathrm{~dB}$ below the time-domain methods. After the third level of perturbation (maximum jitter of $10.2 \%$ ) the deterioration due to jitter in time-domain HNR calculation stabilizes at 21-22 dB, being less representative than shimmer-caused deterioration. The reason for this stabilization is that the HNR within the pulse is not constant. The smallest size of the $\mathrm{N}$ pulses is used as pulse length in the averages (4). Since the synthetic signals consist of exponentially-damped sinusoids, the suppressed portions of the pulses, the endings, are the ones with lower signal energy, resulting in an overestimation of HNR. As the jitter grows the portions removed enlarge, increasing this effect that counters the expected reduction in measured HNR.

When mixed-perturbation signals are used (bottom-right graph in Fig. 3), frequency-domain approaches fail due to their jitter sensitivity, with time-domain methods showing a similar, more acceptable, performance. The effect of pulse length reduction produced by jitter causes the gradual increment of the measured HNR with the level of perturbation.

\section{Conclusions and Future Work}

The correction of the ensemble-average time-domain approach demonstrates to produce satisfactory results using considerably less pulses (4-7) than the original approach (30-50). However, a practical limitation of the correction in (6) has been shown in this work, related to the actual lengths, in samples, of the pulses considered. In general, time domain approaches produced better results than frequency-domain approaches, even with the correction introduced in (10), due to the frequency-domain sensitivity to jitter. This is in correspondence with the predictions in [17].

The results obtained in this paper can serve as a reference regarding error limits in the calculation of Harmonics-to-Noise-Ratios in the presence of different perturbations of the periodicity pattern. However, more extensive experiments can still be performed, including the use of colored and non-uncorrelated noise. Also, other more sophisticated methods could be included in the comparisons, along with a richer set of perturbations levels and combinations. 
Acknowledgments. This work was supported partially by the Canadian International Development Agency Project Tier II-394-TT02-00 and by the Flemish VLIR-UOS Programme for Institutional University Co-operation (IUC).

\section{References}

1. Cox, N. B., Ito, M. R., and Morrison, M. D. Data labeling and sampling effects in harmonics-to-noise ratios. J. Acoust. Soc. Am. 85, 2165-2178. (1989).

2. Cox, N. B., Ito, M. R., and Morrison, M. D. Technical considerations in computation of spectral harmonics-to-noise-ratios for sustained vowels. 32, 203-218. (1989).

3. Ferrer, C. A., González, E. and Hernández-Díaz, M. E. Correcting the use of ensemble averages in the calculation of harmonics to noise ratios in voice signals. J. Acoust. Soc. Am. 118, 605-607. (2005).

4. Fukazawa, T.; El-Assuooty, A. \& Honjo, I. A new index for evaluation of the turbulent noise in pathological voice. Journal of the Acoustical Society of America. Vol. 83, No 3. pp 1189-1193. March. (1988).

5. Hillenbrand, J. A methodological study of perturbation and additive noise in synthetically generated voice signals. J. Speech Hea. Res. 30, 448-461. (1987).

6. Hillenbrand, J., Cleveland, R. A. and Erickson, R. L. Acoustic correlates of breathy vocal quality. J. Speech Hea. Res. 37, 769-778. (1994).

7. Kasuya, H., Ogawa, S., Kazuhiko, M. and Ebihara, S. Normalized noise energy as an acoustic measure to evaluate pathologic voice. J. Acoust. Soc. Am. 80, 1329-1334. (1986).

8. Kojima, H., Gould, W. J., Lambiase, A., and Isshiki, N. Computer analysis of hoarseness. Acta Otolaringol. 89, 547-554. (1980).

9. Lucero, J. C. and Koenig, L. L. Time normalization of voice signals using functional data analysis. J. Acoust. Soc. Am. 108, 1408-1420. (2000).

10. Medan, Y., Yain, E., and Chazan, D. Super resolution pitch determination of the speech signals. IEEE Transactions on Signal Processing. 39, 40-48. (1991).

11. Murphy, P. J. Perturbation-free measurement of harmonics-to-noise ratio in voice signals using pitch-synchronous harmonic analysis. J. Acoust. Soc. Am. 105, 2866-2881. (1999).

12. Parsa, V. and Jamieson, D.G. A comparison of high precision Fo extraction algorithms for sustained vowels. Journal of Speech, Language \& Hearing Research. 42. 112-126. (1999).

13. Qi, Y. Time-normalization in voice analysis. J. Acoust. Soc. Am. 92, 2569-2576. (1992).

14. Qi, Y., Weinberg, B., Bi, N. and Hess, W. J. Minimizing the effect of period determination on the computation of amplitude perturbation in voice. J. Acoust. Soc. Am. 97, 2525-2532. (1995).

15. Qi, Y. and Hillman, R. E. Temporal and spectral estimations of harmonics-to-noise ratio in human voice signals. J. Acoust. Soc. Am. 102, 537-543. (1997).

16. Schoentgen, J., Bensaid, M. and Bucella, F. Multivariate statistical analysis of flat vowel spectra with a view to characterizing dysphonic voices. J. Speech, Lang. Hear. Res. 43, 1493-1508. (2000)

17. Schoentgen, J. Spectral models of additive and modulation noise in speech and phonatory excitation signals. J. Acoust. Soc. Am. 113, 553-562. (2003).

18. Shrivastav, R., and Sapienza, C. M. Objective measures of breathy vocal quality using an auditory model. J. Acoust Soc. Am. 114, 2217-2224. (2003).

19. Titze, I. R. Summary Statement. Workshop-on acoustic voice analysis. National Center of Voice and Speech. Iowa. (1994).

20. Yumoto, E., Gould, W. J., and Baer, T. The harmonics-to-noise ratio as an index of the degree of hoarseness. J. Acoust Soc. Am. 71, 1544-1550. (1982). 